\title{
Direct evidence of dust growth in L183 from mid-infrared light scattering
}

\author{
J. Steinacker ${ }^{1,2}$, L. Pagani ${ }^{1}$, A. Bacmann ${ }^{3}$, and S. Guieu ${ }^{4}$ \\ 1 LERMA \& UMR 8112 du CNRS, Observatoire de Paris, 61 Av. de l'Observatoire, 75014 Paris, France \\ 2 Max-Planck-Institut für Astronomie, Königstuhl 17, 69117 Heidelberg, Germany \\ e-mail: stein@mpia.de \\ 3 Laboratoire d'Astrophysique, Observatoire de Grenoble, BP 53, 38041 Grenoble, Cedex 9, France \\ 4 Spitzer Science Center, 1200 E California Blvd, Mail Stop 220-6, Pasadena CA 91125, USA
}

Received 6 July 2009 / Accepted 30 November 2009

\section{ABSTRACT}

\begin{abstract}
Context. Theoretical arguments suggest that dust grains should grow in the dense cold parts of molecular clouds. Evidence of larger grains has so far been gathered in near/mid infrared extinction and millimeter observations. Interpreting the data is, however, aggravated by the complex interplay of density and dust properties (as well as temperature for thermal emission).

Aims. Direct evidence of larger particles can be derived from scattered mid-infrared (MIR) radiation from a molecular cloud observed in a spectral range where little or no emission from polycyclic aromatic hydrocarbons (PAHs) is expected.

Methods. We present new Spitzer data of L183 in bands that are sensitive and insensitive to PAHs. The visual extinction $A_{\mathrm{V}}$ map derived in a former paper was fitted by a series of 3D Gaussian distributions. For different dust models, we calculate the scattered MIR radiation images of structures that agree with the $A_{\mathrm{V}}$ map and compare them to the Spitzer data.

Results. The Spitzer data of L183 show emission in the 3.6 and $4.5 \mu \mathrm{m}$ bands, while the $5.8 \mu \mathrm{m}$ band shows slight absorption. The emission layer of stochastically heated particles should coincide with the layer of strongest scattering of optical interstellar radiation, which is seen as an outer surface on $I$ band images different from the emission region seen in the Spitzer images. Moreover, PAH emission is expected to strongly increase from 4.5 to $5.8 \mu \mathrm{m}$, which is not seen. Hence, we interpret this emission to be MIR scattered light from grains located further inside the core, and call it "coreshine". Scattered light modeling when assuming interstellar medium dust grains without growth does not reproduce flux measurable by Spitzer. In contrast, models with grains growing with density yield images with a flux and pattern comparable to the Spitzer images in the bands $3.6,4.5$, and $8.0 \mu \mathrm{m}$.

Conclusions. There is direct evidence of dust grain growth in the inner part of L183 from the scattered light MIR images seen by Spitzer.
\end{abstract}

Key words. dust, extinction - ISM: clouds - ISM: individual objects: L183 - infrared: ISM - radiative transfer - scattering

\section{Introduction}

Cold and dense cores of molecular clouds are commonly considered to be a favorable place for the growth of dust particles (see, e.g., Ossenkopf \& Henning 1994). Evidence that grains are larger than commonly observed interstellar medium (ISM) grains have been gathered in different wavelength regions. The thermal emission of dust grains in the far-infrared (FIR) and millimeter $(\mathrm{mm})$ wavelength range shows a change in the index of the spectral density distribution. Based on simple assumptions about the dust opacity law and the spatial and thermal structure, this is interpreted as indirect evidence of larger grains (e.g. Stepnik et al. 2003; Beuther et al. 2004; Ridderstad et al. 2006; Kiss et al. 2006; Schnee et al. 2008). In contrast, Nutter et al. (2008) can model the Taurus Molecular Ring without any assumption about grown particles. In general, disentangling spatial variations in dust properties, density, and temperature is a challenge given the many parameters needed to describe the often clumpy or filamentary cloud structures. This is why, in view of this ambiguity, we call this indirect evidence.

Chapman et al. (2009), McClure (2009), and Chapman \& Mundy (2009) have presented mid-infrared (MIR) extinction curves based on Spitzer and ground-based JHK band observation of stars behind molecular clouds. They find that the extinction curve changes as a function of increasing extinction. McClure (2009) investigates the ice features and concludes that a process involving ice is responsible for the changing shape of the extinction curve. Chapman et al. (2009) find that, with increasing extinction, the extinction law is better fitted by models including larger grains. Similiarly, for the cores investigated in Chapman $\&$ Mundy (2009), the averaged extinction law from 3.6 to $8 \mu \mathrm{m}$ is consistent with a dust model that includes larger grains.

Extinction measurements at optical to MIR wavelengths have the advantage that the temperature of the dust grains no longer influences the results. The disadvantages are (i) the method requires a radiation source like scattered light, diffuse emission from small grains or molecules, or background stellar or extragalactic emission; (ii) the dust extinction properties enter exponentially in the observed extinction of the external radiation so that the source flux, if stellar or extragalactic, must be strong enough to be detectable.

Aside from the thermal emission of the grains and their absorption, they also efficiently scatter radiation. The exploration of the physical properties and spatial distribution of dust particles via the observation of their scattered light was initiated long ago. References to early papers about this topic can be found, e.g., in Sandell \& Mattila (1975). Common ISM grains with sizes around $0.1 \mu \mathrm{m}$ efficiently scatter optical and near-infrared 
(NIR) radiation so that images observed at these wavelengths can be used to study the spatial structure of the dust, as well as its properties. This method has widely been used in star formation research, e.g. to analyze the spatial 3D structure of circumstellar disk candidates (see, e.g., Steinacker et al. 2006). Nakajima et al. (2003) interpret the surface brightness seen in $J, H$, and $K$ s images of the Lupus 3 dark cloud as starlight scattered by dust. Foster \& Goodman (2006) propose to use this "cloudshine" as a general tracer of the column density of dense clouds and presented NIR scattered light images of the Perseus molecular cloud complex. Padoan et al. (2006) present a corresponding method and test it by calculating NIR scattered light images from the results of magnetohydrodynamic turbulence simulations and by comparing the resulting column density distributions with the original simulation data. They argue that the method can be applied to filamentary cloud structures being illuminated by a homogenous radiation field in the range of visual extinction between 1 and 20 mag. The NIR cloud shine of a Corona Australis cloud filament was investigated in this way by Juvela et al. (2008).

It seems promising to observe scattered light from cores at longer wavelengths, because the lower extinction allows the inner parts to be traced. However, the scattering cross section of the ISM dust particles drops with wavelength once the wavelength is much larger than the particle size. Scattered light from cores at MIR wavelengths therefore has not been reported so far and has been overlooked until now despite submillimeter observations often being explained by grain growth to sizes for which MIR scattering may become visible. Additionally, polycyclic aromatic hydrocarbon (PAH) emission starts to dominate the emission pattern, at most MIR wavelengths, preventing the identification of scattered light.

It turns out that indeed the scattered light image analysis at MIR wavelengths opens up a window to trace bigger grains: (i) they scatter radiation efficiently at wavelengths for which the smaller grains already enter the regime of Rayleigh scattering where absorption dominates scattering; (ii) the optical depth is reduced compared to the depth at NIR wavelengths so that denser regions can be traced with a better chance of seeing grown particles.

Observations with the Spitzer telescope allow us to trace the relevant wavelengths and to check the existence of possible $\mathrm{PAH}$ emission by using a combination of filters, some including strong emission features and some excluding all. Nearby starless low- to mean-mass clouds are potential candidates for observing this MIR scattered light which we will call "coreshine" as it is produced in layers of high densities near the central core or cores, in contrast to the cloudshine seen in the low-density cloud parts.

L183 (also known as L134N) is such a starless dark cloud high above the Galactic plane $\left(36^{\circ}\right)$, hence close to us $(110 \mathrm{pc}$, Franco 1989). Its mass has been estimated to $\sim 80 M_{\odot}$ (Pagani et al. 2004, hereafter Paper I). It contains an elongated ridge of dense material with two prestellar cores and the main prestellar core has a peak extinction of $A_{\mathrm{V}} \approx 150$ mag with a dust temperature close to $7 \mathrm{~K}$ (Paper I). It was subsequently shown that the gas is thermalized with the dust in the core (Pagani et al. 2007).

The Spitzer observations of L183 are summarized in Sect. 2. In Sect. 3, we fit the $A_{\mathrm{V}}$ map presented in Paper I with a series of $3 \mathrm{D}$ density basis functions. The results of a scattered light image modeling are presented in Sect. 4 based on a dust growth model and compared to the different Spitzer images of L183. In Sect. 5, we summarize our findings.

\section{Spitzer observations of L183}

To describe different parts of the cloud, we refer to the $A_{\mathrm{V}}$-map presented in Paper I (see their Fig. 1 for a detailed comparison), although it is a projected view on the spatial cloud structure. Throughout this paper, we use the term outer cloud region for the parts of the L183 cloud where $A_{\mathrm{V}}<15$, (banana-shaped) central part for $15<A_{\mathrm{V}}<90$, and central core for the densest region with $A_{\mathrm{V}}>90$, respectively.

The Spitzer IRAC (3.6 to $8 \mu \mathrm{m}$, Fazio et al. 2004) observations of L183 were obtained as part of the Cycle 1 GTO program 94 (PI: Charles Lawrence). The observations were broken into two epochs, the astronomical observation requests (AORs) are both centered at $15^{\mathrm{h}} 54^{\mathrm{m}} 15.00^{\mathrm{s}}-2^{\mathrm{d}} 55^{\mathrm{m}} 0.0^{\mathrm{s}}$, they were obtained on 23 and 25 of August 2005, and the AORKEYs are 4921600 and 4921856 in chronological order. Each AOR was constructed with the same strategy: at each map step, three dither positions were observed, each with high-dynamic-range exposures of 1.2 and $30 \mathrm{~s}$.

To build the full mosaics of L183, we started with the Spitzer Science Center (SSC) pipeline-produced, basic calibrated data (BCDs), version S14.0. We ran the IRAC artifact mitigation code written by Carey and available on the SSC website. We constructed a mosaic for each epoch from the corrected BCDs using the SSC mosaicking and point-source extraction (MOPEX) software (Makovoz \& Marleau 2005), with a pixel size of $1.22^{\prime \prime} \mathrm{px}^{-1}$, very close to the native pixel scale.

The inner parts of 3.6, 4.5, and $8 \mu \mathrm{m}$ mosaics are shown in Fig. 1. One can clearly see a banana-shaped structure (see also Sect. 4.2) visible in emission at 3.6 and $4.5 \mu \mathrm{m}$ and in absorption at $8 \mu \mathrm{m}$. The spatial structure resembles well the shape of the densest region of the cloud complex visible in the $A_{\mathrm{V}}$ map presented in Paper I. The $5.8 \mu \mathrm{m}$ image shows horizontal stripes and a strong vertical gradient in signal-to-noise ratio $(\mathrm{S} / \mathrm{N})$ so that only horizontal pieces of the upper part of the image contain reliable information on the flux. For this reason, we do not show the image here, and do not include it in the image modeling. The upper part of the central banana-shaped structures is seen in absorption in the reliable stripes.

To show the magnitude of the emission and absorption features, we present in Fig. 2 cuts of the IRAC maps through the central core (declination $-2^{\circ} 52^{\prime} 48^{\prime \prime}$ ) seen in absorption on all images. As the cut lies in a stripe of the $5.8 \mu \mathrm{m}$ image with an acceptable $\mathrm{S} / \mathrm{N}$, we also include the flux in this band. The gray thin lines indicate an averaged external flux. From the cuts, the increase by almost two orders of magnitude in the external flux is visible when changing from 3.6 to $8 \mu \mathrm{m}$.

The thermal emission of dust particles which are large enough to be considered as black body emitters cannot produce a measurable flux given the low temperature of a few ten $\mathrm{K}$ (Pagani et al. 2004). Even if all dust particles in L183 would have a temperature of $40 \mathrm{~K}$, the specific flux density measured on Earth at $\lambda=8 \mu \mathrm{m}$ would be $<7 \times 10^{-29} \mathrm{Jy} / \mathrm{sr}$ (assuming $0.1 \mu \mathrm{m}$-sized grains and a gas-to-dust mass ratio of 100), and lower for the shorter IRAC band wavelengths.

The emission in the Spitzer wavelength range could also be caused by smaller particles that are stochastically heated like PAHs. Figure 3 illustrates the presence of PAH emission features in IRAC bands by showing the instrumental response of IRAC (gray areas) for the different filters as a function of wavelength. The transmission of radiation through a layer of dust is plotted as a black dashed line (see Draine 2003a,b,c). The light-gray (red in the electronic version) line shows the expected location of PAH features (Draine \& Li 2007). 


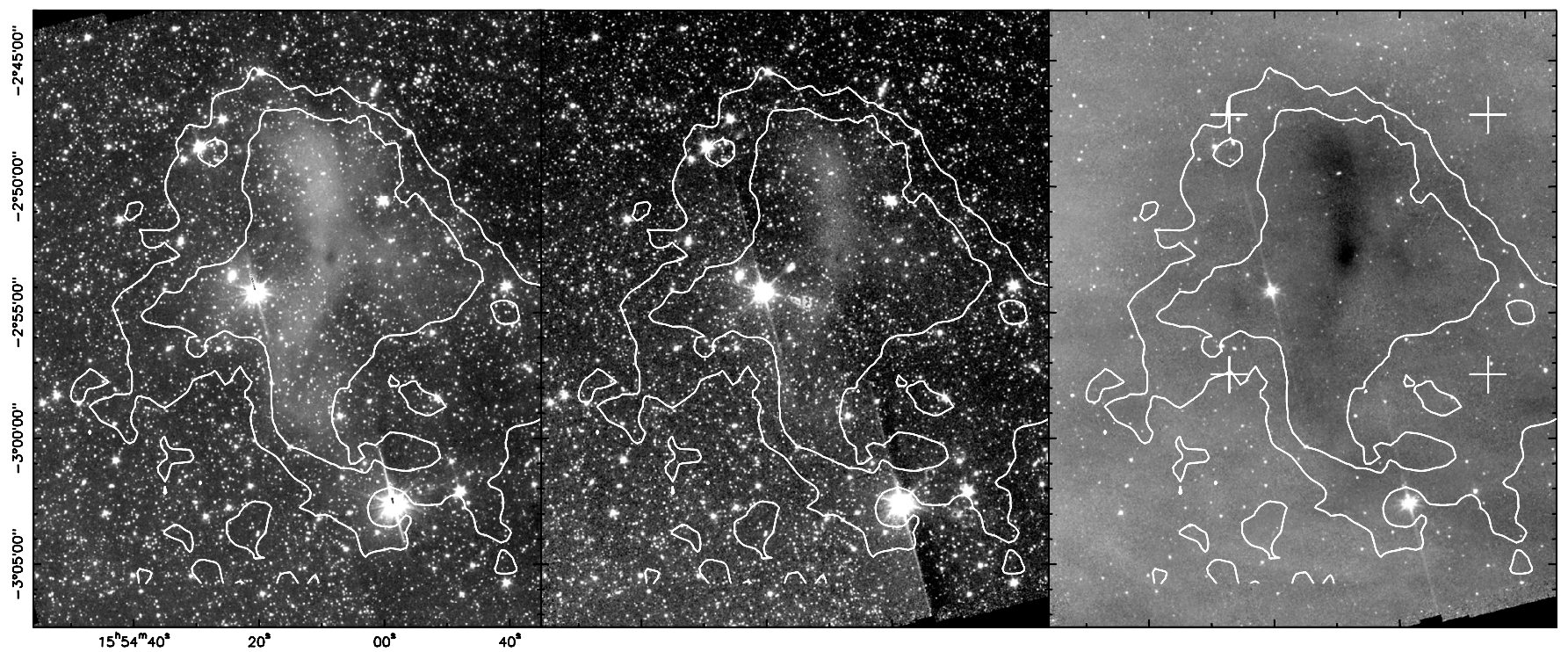

Fig. 1. IRAC 3.6, 4.5, and $8 \mu \mathrm{m}$ long exposure mosaics. Superposed on each image are the $A_{\mathrm{V}}=5$ and 10 mag contours from the $A_{\mathrm{V}}$ map of Paper I. The white crosses in the $8 \mu \mathrm{m}$ image mark the region that has been modeled in this paper.

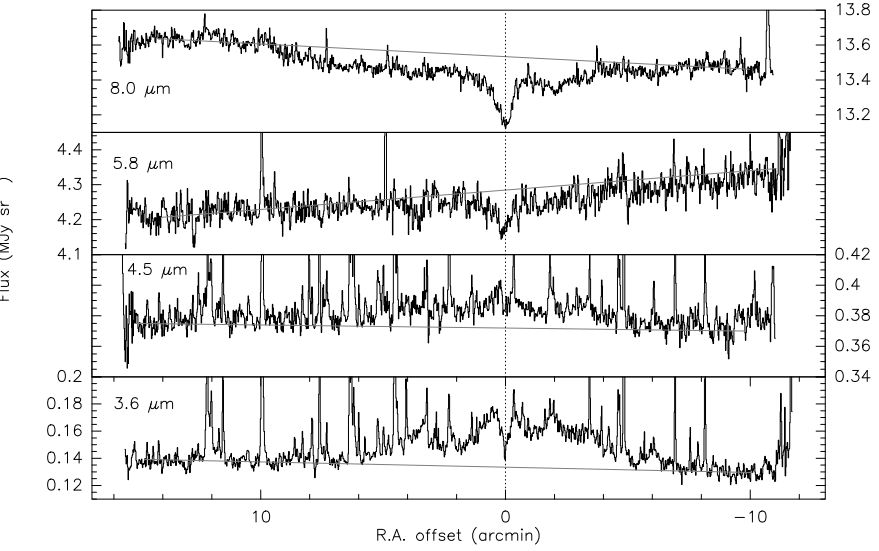

Fig. 2. Cuts from the IRAC maps (Fig. 1) at constant declination $\left(-2^{\circ} 52^{\prime} 48^{\prime \prime}\right)$ through the dust peak in all 4 bands. The dotted line indicates the position of the dust peak. Excess emission is seen at 3.6 and $4.5 \mu \mathrm{m}$ while absorption dominates for the 5.8 and $8.0 \mu \mathrm{m}$ ranges.

Explaining the observed emission in the 3.6 and $4.5 \mu \mathrm{m}$ bands as the emission of stochastically heated particles (PAHs or small dust grains) is difficult for two reasons. First, PAHs and small grains need an exciting UV to optical radiation field. As dust particles have the same order of magnitude in absorption and scattering cross section at these wavelengths, the scattered light images at optical wavelength give a hint of the morphology of the layer where most of the outer exciting UV to optical radiation is absorbed. As seen, e.g, in Fig. 4d of Juvela et al. (2002), this layer resembles the shape of the $A_{\mathrm{V}}=1$-layer in the $A_{\mathrm{V}}$ map closely (Paper I). In contrast, the emission pattern resembles the shape of the inner banana-like structure while no emission can be seen with the shape of the outer layer. Even if holes would make it possible for exciting radiation to reach inner parts, a substantial part of the PAH emission should come from the outer layer which is not what is seen.

For completeness, we also investigated the possibility of holes. We performed detailed 3D ray-tracing for a large sample of structures that are consistent with the column density

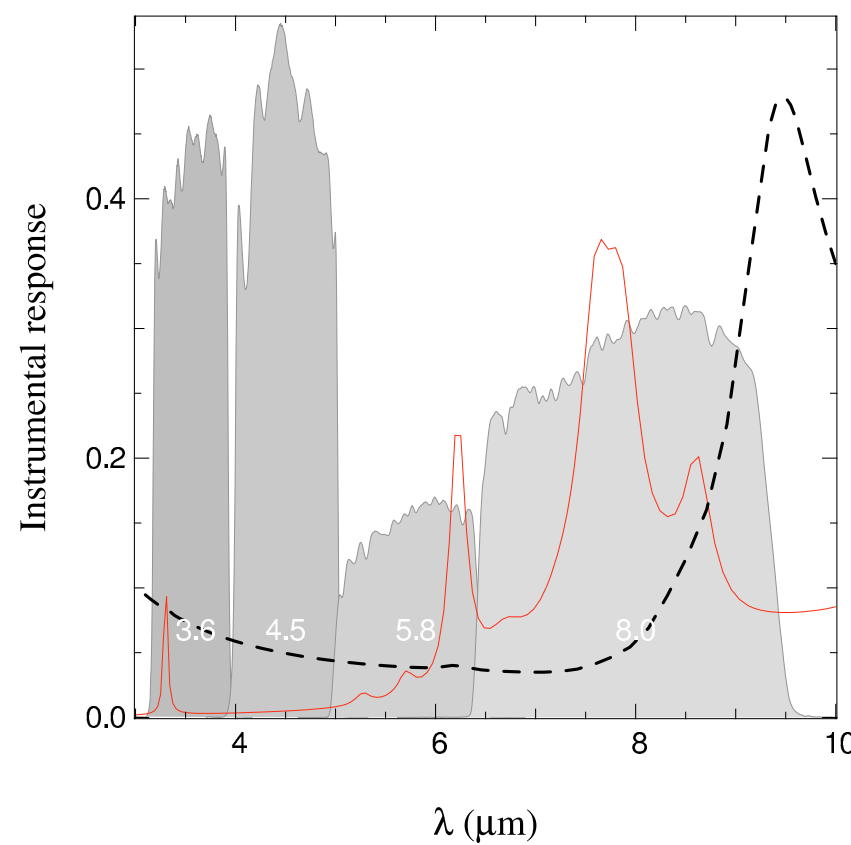

Fig. 3. The gray areas are the instrumental response of each IRAC filters as a function of the wavelength. The light gray (red in the electronic version) line is a model of infrared emission from dust grains by Draine $\& \operatorname{Li}(2007)\left(q \_\mathrm{PAH}=4.58 \% U=1\right)$. The black dashed line is a model of the dust extinction from Draine (see 2003a,b,c). The models are unitless, and have been scaled to fit in the figure.

information contained in the $A_{\mathrm{V}}$ map. The results are presented in Appendix A. Based on 3D spatial structure variations and the analysis of $\tau$-iso-surfaces, we conclude that only a small fraction of all spatial structure models would allow for PAH emission near the central absorption peak. It therefore seems unlikely that the cloud complex has one of these rare configurations with a hole deep enough to let the interstellar radiation field (ISRF) reach the central part so that PAHs could be excited to produce the observed inner emission. Moreover, the illumination through 
a single hole would not produce a smooth, even illumination of the entire inner structure as seen in the $3.6 \mu \mathrm{m}$ image.

Second, based on an integration of the gray/red curve in Fig. 3, the PAH emission in the $5.8 \mu \mathrm{m}$ band should be a factor of about 10 stronger than the emission in the $4.5 \mu \mathrm{m}$ band. The improved dust transmission in the $5.8 \mu \mathrm{m}$ band will amplify this effect. In turn, the external radiation at $5.8 \mu \mathrm{m}$ is about a factor of 10 stronger than at $4.5 \mu \mathrm{m}$. Based on Flagey et al. (2006), we estimated the flux one would expect if the excess flux at $4.5 \mu \mathrm{m}$ was due to PAHs. We found that, in all reasonable cases, the $5.8 \mu \mathrm{m}$ flux should be seen in emission (about 1 to 2 orders of magnitude stronger than at $4.5 \mu \mathrm{m}$ ) and not in absorption as presently observed (see cuts in Fig. 2).

These two arguments suggest that emission from stochastically heated grains likely fails as an explanation of the emission structure. We therefore explore in this paper whether the emission seen in the 3.6 and $4.5 \mu \mathrm{m}$ filters can be interpreted as stellar light scattered by the dust particles in the cloud gas, and what dust properties would be needed to qualitatively reproduce the images.

\section{Av map modeling}

Extinction maps average the 3D density distribution of the molecular cloud over the line of sight (LoS). This adds an important source of ambiguity to the interpretation of the extinction map: each observed extinction value in the plane of sky agrees with a whole range of density distributions and dust properties. As a result, another source of information is needed to remove this ambiguity. This can be, e.g., a measurement at FIR/mm wavelengths. Multi-wavelength modeling of images from molecular clouds and cloud cores incorporating the hidden third dimension has not been used widely so far, but is possible in the mean time (see, e.g., Steinacker et al. 2005). Another source of information are scattered light images. They depend on the 3D spatial structure of the cloud, on the dust properties, and the external $3 \mathrm{D}$ radiation field.

In this work, we combine the information about L183 from the extinction map with the scattered light images obtained by Spitzer. To obtain a 3D dust density data cube required for the scattering calculations, we fit the available $A_{\mathrm{V}}$ map of L183 (Paper I) with a series of density basis functions flexible enough to deal with the complex spatial structure of the cloud. The fit of the $2 \mathrm{D} A_{\mathrm{V}}$ map is done by determining the optical depth along the LoS through a 3D density structure described by the series. This means that parameters describing the spatial structure along the line of sight will not be determined by this fit. Instead, a library of possible spatial structures is created and used for the modeling. Details of the technique to fit the $A_{\mathrm{V}}$ map are described in Steinacker et al. (2005), but here we just summarize the basic approach and refer readers to that paper for details. The dust properties are fixed for this determination to $A=0.1 \mu \mathrm{m}$ silicate spheres, but in later sections we re-interpret the obtained optical depths using other grain sizes.

The underlying 3D density structure is described by a series of 100 3D Gaussian density clumps with their main axes aligned with the Cartesian coordinate axes. The position and extent in the plane of sky of each Gaussian (4 parameters) and their normalization factor (5th parameter) are optimized with a special simulated annealing search tool (Thamm et al. 1994). The high number of Gaussians is necessary because the $A_{\mathrm{V}}$ map is very clumpy with many local maxima and structure on small and large scales. Tests with lower numbers of Gaussians could not reproduce the

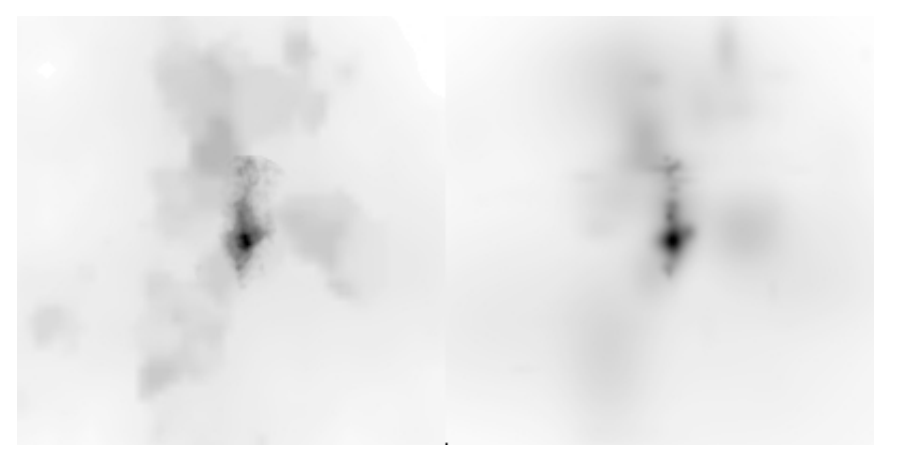

Fig. 4. Inner $66000 \mathrm{AU}$ of the $A_{\mathrm{V}}$ map from Paper I (left) and the fitted map using 3D Gaussian clumps (right).

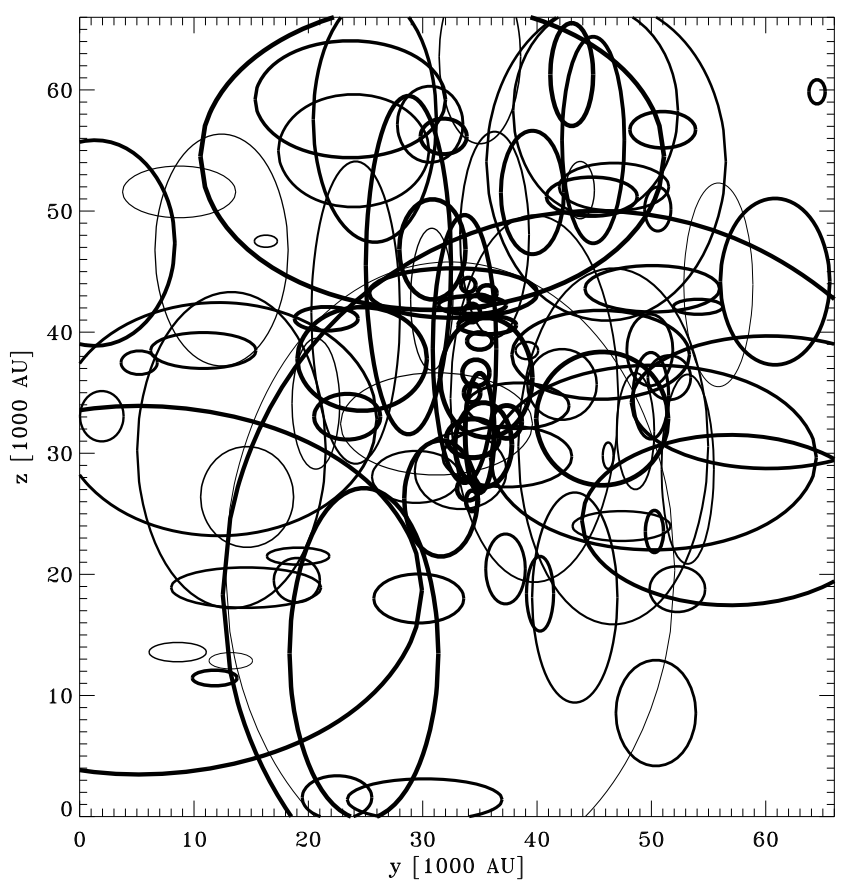

Fig. 5. Position and main axes of the Gaussian basis functions used to fit the inner part of the $A_{\mathrm{V}}$ map. The coordinates $x$ and $y$ are in the plane of sky.

main features of the map adequately. It must be stressed, however, that the original map in Paper I is incomplete (lower limit to the extinction toward the northern prestellar core) and that it probably suffers from aliasing in the intermediate opacity range ( $\left.A_{\mathrm{V}}=20-40 \mathrm{mag}\right)$ because there are few background objects.

In Fig. 4, we show the inner $66000 \mathrm{AU}$ of the $A_{\mathrm{V}}$ map (left) and the corresponding fitted map using Gaussian clumps (right). The underlying Gaussian distribution is sketched in Fig. 5 by showing ellipses with the main axes of the Gaussians at their corresponding position in the plane of sky. The line thickness is a measure of the mass in the different clumps. The figure shows that the clumpy structure of the $A_{\mathrm{V}}$ map requires a series of basis functions in the inner $10000 \mathrm{AU}$ along the $x$-axis to reproduce the bananashape of the cloud center. In turn, some of the detailed spatial structures (e.g. in the lower left part of the $A_{\mathrm{V}}$ map) are represented by a low number of basis functions, which indicates that the chosen number of 100 is still not enough to describe all features of the map.

The fit of the $A_{\mathrm{V}}$ map provides us with a column density structure, but the extent and position of the Gaussians along the line of sight are still unknown. To provide a basis for the fits of 

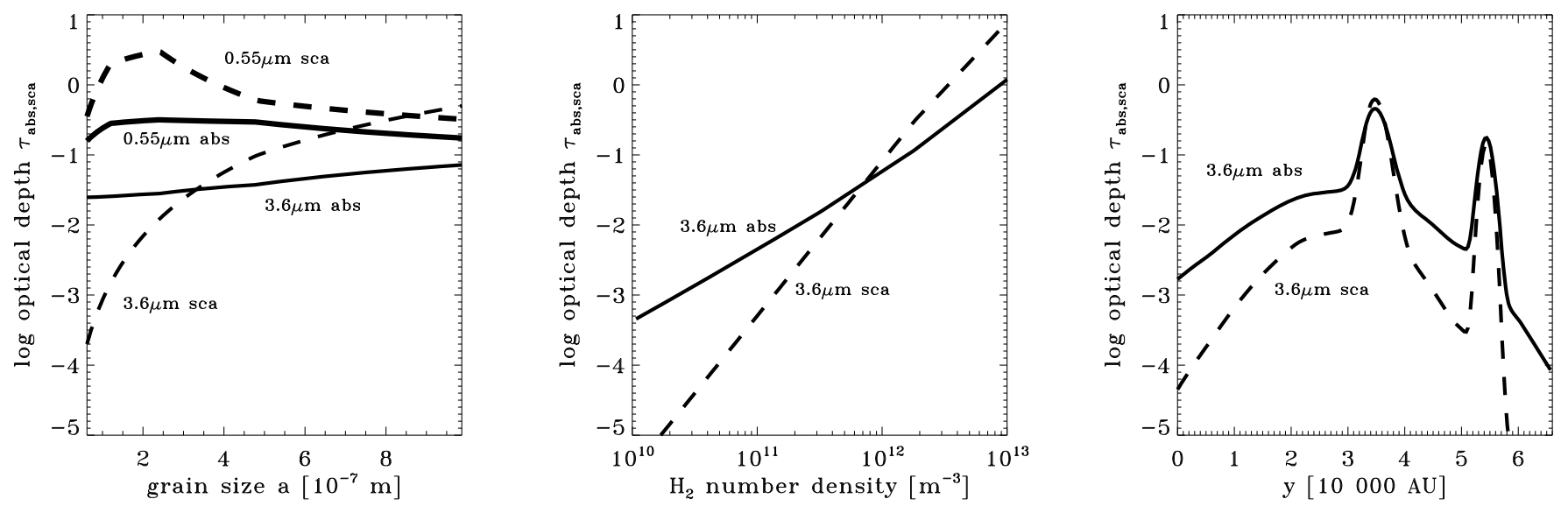

Fig. 6. Left: local optical depth in a cell of the 3D density structure (constant density) as a function of the grain size (assuming dust mass conservation). The solid (dashed) line refers to absorption (scattering), and the thick (thin) line to a wavelength of $0.55 \mu \mathrm{m}(3.6 \mu \mathrm{m})$. Middle: optical depth for dust following Eq. (1) as a function of the cell gas number density for absorption and scattering at $\lambda=3.6 \mu \mathrm{m}\left(a_{0}=6 \times 10^{-8} \mathrm{~m}, \alpha=0.4\right.$, $n_{\mathrm{H}_{2} ; 0}=10^{10} \mathrm{~m}^{-3}$ ). Right: local absorption and scattering optical depth along a typical ray crossing one of the $100003 \mathrm{D}$ density data cubes that fit the $A_{\mathrm{V}}$ map.

the scattered light images, we have created a library of 10000 3D density structures where the spatial structure parameters along the LoS were chosen randomly given the outer physical borders and the constraint to agree with the fit of the $A_{\mathrm{V}}$ map as shown in Fig. 4.

\section{Scattered light image modeling using a dust growth model}

\subsection{Dust model}

It is expected that in the cold core environment, the particles grow to fluffy aggregates, vary in their chemical composition and follow an entire size distribution (see, e.g., Ossenkopf 1993). While there are no direct measurements of the dust grain size distribution in dense cores, model calculations and laboratory experiments at least can provide rough estimates of the mean grain size as a function of the gas number density in one free-fall time (Flower et al. 2005). Therefore, we assume that the grains have properties approximately described by spherical homogeneous particles of one size and chemical composition. The radius of the grains $a$ is assumed as a simple powerlaw of the $\mathrm{H}_{2}$ number density $n_{\mathrm{H}_{2}}$ with the spectral index $\alpha$

$a= \begin{cases}a_{0}\left(\frac{n_{\mathrm{H}_{2}}}{n_{\mathrm{H}_{2} ; 0}}\right)^{\alpha} & n_{\mathrm{H}_{2}}>n_{\mathrm{H}_{2} ; 0} \\ a_{0} & n_{\mathrm{H}_{2}} \leq n_{\mathrm{H}_{2} ; 0}\end{cases}$

so that the grains in regions below the threshold density $n_{\mathrm{H}_{2} ; 0}$ have the size $a_{0}$. The calculations leading to the best-fitting results described in this section were performed with varying values for $a_{0}, n_{\mathrm{H}_{2} ; 0}$, and $\alpha$. Poppe \& Blum (1997), e.g., found a growth index of $\alpha=0.25$ (see Flower et al. 2005, their Fig. 3, plotting the dependence in double-logarithmic scale).

In Sect. 3, we modeled the local optical depth for extinction $\tau_{\text {ext }}(\lambda)$ for $\lambda=0.55 \mu \mathrm{m}$ toward the observer for each cell of our spatial grid. For the radiative transfer modeling of the Spitzer scattered light images, we need to calculate the optical depth for absorption and scattering at the Spitzer band wavelengths in each cell. If we know the grain size and the number density of these grains, $n$, in each cell, we can use existing opacity tables to find the cross sections $\sigma_{\mathrm{abs} / \mathrm{sca}}(\lambda, a)$ and calculate the optical depths.
To use Eq. (1), this requires finding the gas density from the derived local extinction $\tau_{\text {ext }}(0.55 \mu \mathrm{m})$.

The optical depth across a Cartesian grid cell with the edge length $s$ is

$\tau_{\text {ext }}(0.55 \mu \mathrm{m})=\sigma_{\text {ext }}(0.55 \mu \mathrm{m}, A) n_{0} s$,

if all particles have the size $A$ and $n_{0}=n(A)$. Assuming an internal density of each homogeneous grain $\rho_{\mathrm{d}}$, the mass of a grain is $m_{\mathrm{d}}=4 \pi A^{3} \rho_{\mathrm{d}} / 3$, and the total dust mass in the cell volume is $n_{0} m_{\mathrm{d}} s^{3}$. For a given mass gas-to-dust ratio $R_{\mathrm{g} / \mathrm{d}}$, this defines the $\mathrm{H}_{2}$ mass $n_{\mathrm{H}_{2}} m_{\mathrm{H}_{2}} s^{3}$, hence

$n_{\mathrm{H}_{2}}=\frac{4 \pi A^{3} \rho_{\mathrm{d}}}{3 m_{\mathrm{H}_{2}}} R_{\mathrm{g} / \mathrm{d}} \frac{\tau_{\mathrm{ext}}(0.55 \mu \mathrm{m})}{\sigma_{\mathrm{ext}}(0.55 \mu \mathrm{m}, A)} \frac{1}{\mathrm{~s}}$.

This allows us to calculate the $\mathrm{H}_{2}$ densities for all cells and the corresponding grain size using Eq. (1). The corresponding number density $n(a)=n_{0} A^{3} a^{-3}$ results from the conservation of dust mass in the cell. Using the Draine \& Lee (1984) opacities, we can then calculate the corresponding local MIR optical depth $\tau=\sigma_{\mathrm{abs} / \mathrm{sca}}(3.6 \mu \mathrm{m}, a) n(a) s$ for the absorption and scattering needed for the radiative transfer modeling of the scattered light images.

The effect of grain growth on optical depth due to absorption and scattering is illustrated in Fig. 6. The left plot shows the optical depth for absorption and scattering through a typical cell of the 3D density structure with constant density for $\lambda=0.55 \mu \mathrm{m}$ and $3.6 \mu \mathrm{m}$ as a function of the grain size. The grain numbers have been chosen to conserve the dust mass in the cell. For optical wavelengths, the change in the ratio of $\sigma_{\text {abs }}$ and $\sigma_{\text {sca }}$ is less than a factor of 4 over a tenfold increase in size. In contrast, at $3.6 \mu \mathrm{m}$, absorption becomes more efficient by a factor of 3 , while scattering is amplified by a factor of 300 . Even a moderate increase of grain size by a factor of 2 will result in about 10 times more efficient scattering. Correspondingly, the MIR wavelength range allows to see larger grains in scattered light as scattering becomes efficient again for higher gas number densities above about $n_{\mathrm{H}_{2} ; 0}$ if the optical depth for absorption allows the scattered light to proceed toward the observer. The dependence of the optical depth for absorption and scattering on the gas number density when using Eq. (1) is illustrated in the middle figure. In the densest regions, the optical depth for scattering is larger 
than for absorption at $3.6 \mu \mathrm{m}$. The right plot gives the optical depths for a typical ray cutting an entire density cube consistent with the $A_{\mathrm{V}}$ map of L183 and including grain growth. In this figure we see that while we cross clumps of high density, scattering becomes stronger, so that scattered light should be seen near the densest regions in contrast to NIR light, for which more scattering is seen in the outer parts. Whether scattered light indeed reaches the observer from the inner parts is a complex interplay of background radiation, optical depth, and forward scattering, and can only be answered by running a radiative transfer code.

Aside from the change in the scattering efficiency due to the dust particle growth, the scattering phase function describing how radiation is scattered from one direction to another also changes. Figure 7 shows a typical phase function in polar coordinates of a homogeneous spherical silicate particle at a wavelength of $3.6 \mu \mathrm{m}$ (Steinacker et al. 2003) placed at the origin. While small ISM particles scatter almost isotropically at this wavelength, the scattering becomes peaked in the forward direction for larger grains.

This forward beaming can alter or even dominate the appearance of the scattering images. For the outer region of the core, the interstellar radiation field (ISRF) reaches the dust from many directions and is scattered almost isotropically. For the central region, mainly forward scattering dominates because of grown particles. The interstellar radiation from the direction of the observer will not be scattered in a backward direction. Radiation hitting the core in the plane of the sky also has a low efficiency to be scattered by $90^{\circ}$. Only radiation from the background is scattered efficiently in a forward direction toward the observer. But this radiation has to pass the central parts of the cloud first and will be seen only if the optical depth is still so little that emerging flux can be detected. For Spitzer, this might actually limit the possibilities of observing the growth of grains in the regions of highest density. However, with the sensitivity of the midinfrared instrument onboard the James Webb Space Telescope, the wavelength range $>5 \mu \mathrm{m}$ will become important for tracing larger grains.

\subsection{Theoretical core shine images of $L 183$ and dust-modeling results}

The presentation of the image modeling results in this section has two goals: (i) to demonstrate that scattered light images based on a density structure in agreement with the current extinction data of L183 can reproduce the main features of the observed Spitzer images; and (ii) to present evidence that only models with grain growth can reproduce the appearance of the central banana-shaped region in contrast to models with ISM grains without growth.

For the first goal, it is sufficient to present one image with a distinct set of parameters of the density structure model, the dust data, and the external field that shows overall agreement. The common approach for the second goal would be an automated fit and exploration of the parameter space quantifying the agreement on the basis of a $\chi^{2}$-minimization as we used it for the $A_{\mathrm{V}}$-fit. An automated fitting procedure varying the many free parameters is prohibitive, though as radiative transfer calculations applied to a clumpy 3D structure like L183 including scattering are time-consuming. For the images shown in this section, we used $50^{3}$ spatial grid cells and a discretization of the direction space in 648 directions leading to a intensity solution vector with $8.1 \times 10^{7}$ values. An automated fitting procedure would need to repeat the calculation of this vector at least several ten thousand times to optimize the density structure.

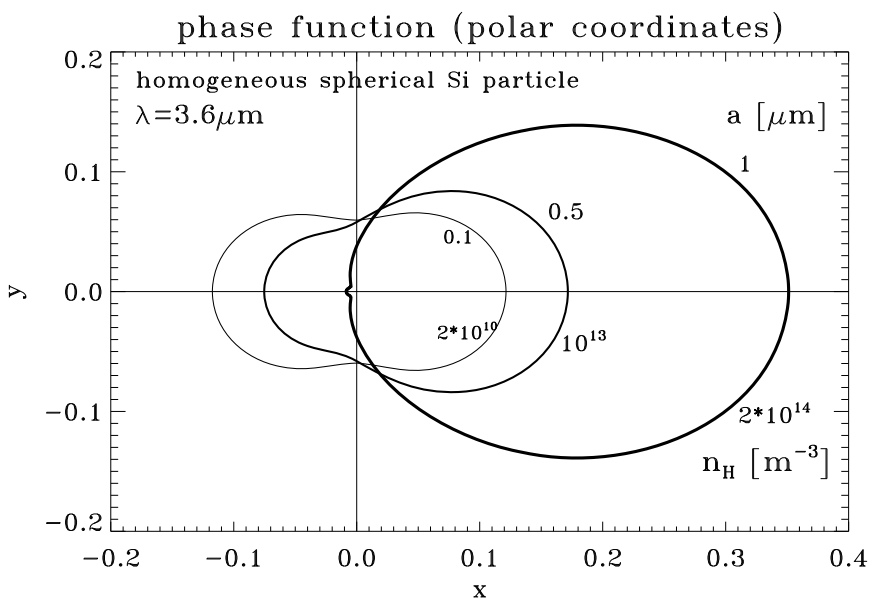

Fig. 7. Phase function in polar coordinates of a homogeneous spherical silicate particle for different particle sizes $a$ at a wavelength of $3.6 \mu \mathrm{m}$. The $\mathrm{H}_{2}$ number densities corresponding to each $a$ as defined in Eq. (1) are given in the lower right part.

Therefore, we present here the results of a nonautomated exploration based on the 10000 spatial structures derived from the $A_{\mathrm{V}}$ map fitting as a representation of possible density distributions of the cloud. The quality of the fit was decided by eye: reproduction of the central banana-shaped region and its maximum in the northern part, the radiation depression in the central core, the overall agreement with the flux level observed with Spitzer, and the shape and location of the weak emission patterns in the outer region.

Using the ray-tracing version of our 3D radiative transfer code (Steinacker et al. 2003), we selected spatial structures, which yield images fitting the Spitzer $3.6 \mu \mathrm{m}$ image for varying dust models. Then we further changed both the structure and the dust parameters by hand to study the influence of each parameter and to find the best-fitting parameter set. Finally, we calculated the images at 4.5 and $8.0 \mu \mathrm{m}$ for the same parameter set.

As an external radiation field, we used the DIRBE data provided by the legacy archive microwave background data analysis $\left(\right.$ LAMBDA $^{1}$ ) to take the global anisotropy of the ISRF into account. Moreover, a nearby star could be another (local) source of anisotropic illumination. Investigating the stars in the vicinity of L183, we found one star that could be near or in the cloud. In Appendix B, we discuss the spectral energy distribution (SED), and the resulting stellar properties. A star consistent with the SED and placed near or even in the outer parts of L183 would locally influence the scattered light image at $3.6 \mu \mathrm{m}$ up to distances of $2000 \mathrm{AU}$ from the star. In all other parts of L183, the ISRF dominates the radiation field. Therefore, we left the star out in our consideration, like the other stars visible in the IRAC images.

Figure 8 compares the Spitzer images with the resulting scattered light model images. The upper images show the inner $66000 \mathrm{AU}$ of the cloud at 3.6, 4.5, and $8 \mu \mathrm{m}$ as observed by Spitzer (see Fig. 1). Images calculated without growth of the grains were too low in scattered light flux at $3.6 \mu \mathrm{m}$ for various ISM grain sizes, and all fluxes were below $10^{-5} \mathrm{MJy} / \mathrm{sr}$. The lower images show the best-fitting model images at 3.6 (left), 4.5 (middle), and $8.0 \mu \mathrm{m}$. The corresponding parameters of the growth model as described by Eq. (1) are $\alpha=0.4$ for the powerlaw index, a grain size in the outer region of $a_{0}=5 \times 10^{-8} \mathrm{~m}$, and $\mathrm{H}_{2}$ number density threshold for growth at $3 \times 10^{10} \mathrm{~m}^{-3}$.

1 http://lambda.gsfc.nasa.gov 
3.6 micron

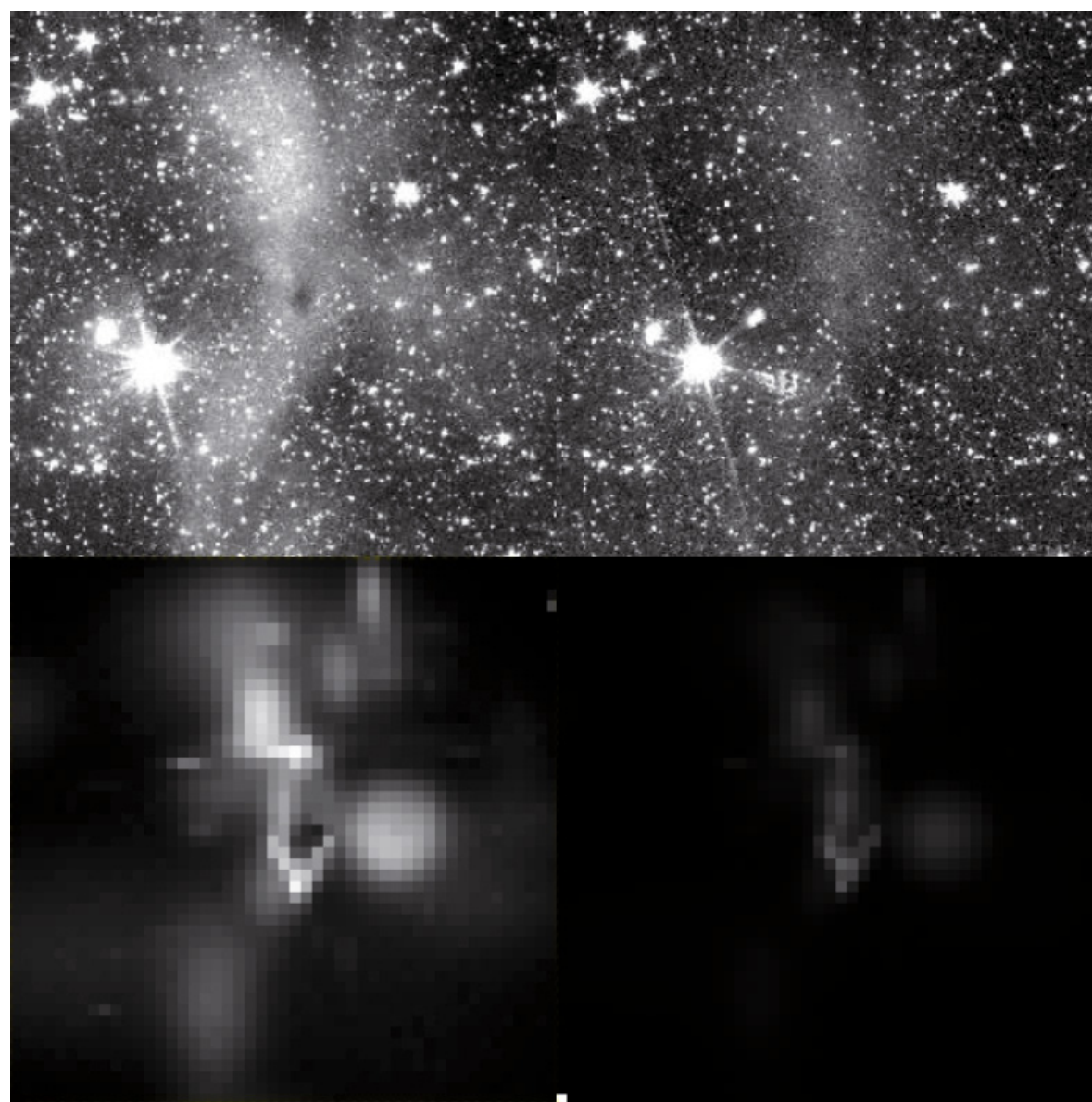

\section{0 micron}

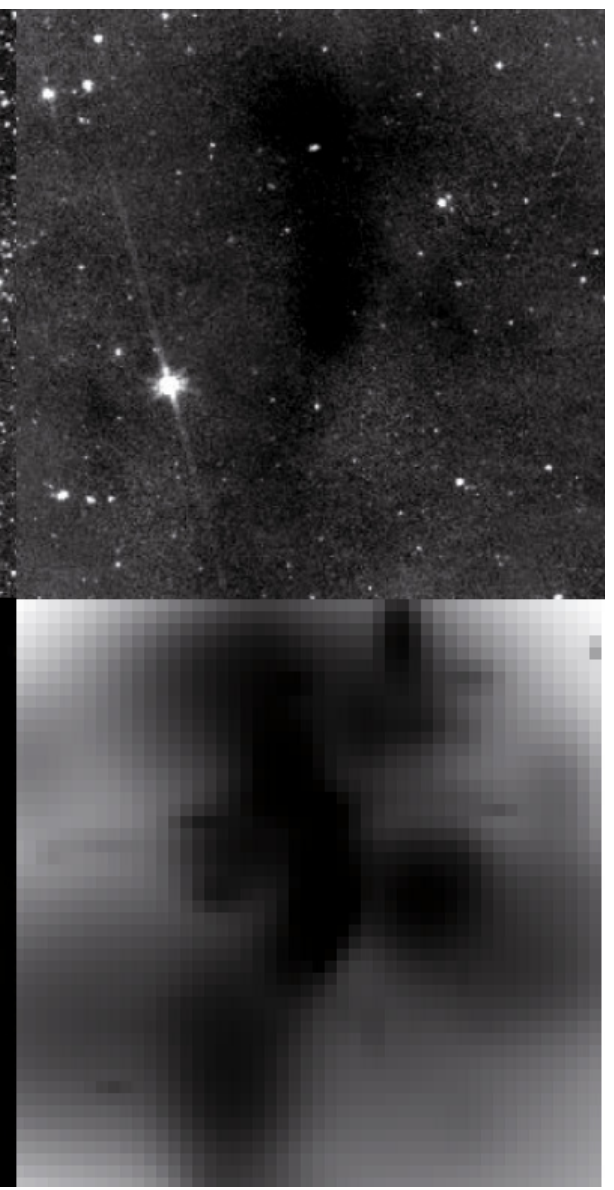

Fig. 8. Comparison of the three Spitzer images at 3.6, 4.5, and $8 \mu \mathrm{m}$ of the inner $66000 \mathrm{AU}$ of L183 (top) with scattered light models based on grains growing as a function of density (bottom). The underlying 3D structure of the model images is consistent with the measured $A_{\mathrm{V}}$ map. The general pattern of the modeled diffuse emission is similiar while, the flux is about a factor of 2 lower in the model.

In the lower left hand image, the overall emission pattern is well-matched with more emission in the northern part. The model images show some peaks (e.g. directly below the central core), which obviously are not present in the Spitzer images. Investigating the underlying density structure, we found that those are attributed to single Gaussian clumps and the image peaks could not be removed by changing their LoS position or extent. We relate them to either a remaining error in fitting the $A_{\mathrm{V}}$ map or to uncertainties in the $A_{\mathrm{V}}$ map itself.

The lower middle $4.5 \mu \mathrm{m}$ image also shows overall agreement with the corresponding Spitzer image. The same flux normalization is used as in the $3.6 \mu \mathrm{m}$ image. The upper part of the central banana-shaped region is visible, and the flux ratio in the observed and theoretical images $F_{4.5 \mu \mathrm{m} / 3.6 \mu \mathrm{m}}$ is about 0.2 . The $3.6 \mu \mathrm{m}$ Spitzer image shows the central core in absorption and also in the $4.5 \mu \mathrm{m}$ image this dip is weakly seen (compare with Fig. 2). In the model, the dip is reproduced at both wavelengths. The actual size of the dip is not reproduced because of the limited size of the computational cells.

For the $8 \mu \mathrm{m}$ image, a background flux value was interpolated from regions of low extinction in the Spitzer image and assumed as a constant background. Therefore, comparing the image with the Spitzer data has to be done with caution: bright regions in the lower right image correspond to regions where the background radiation experiences little extinction. The calculations include scattering (which is very low even in the presence of larger grains), but do not consider the effect of PAH emission by the cloud itself.

As absorption dominates at all densities, the image reproduces the main features of the $A_{\mathrm{V}}$ map. It also reveals where the density structure model was still too coarse: single Gaussian clumps appear where the Spitzer image shows more complex absorption structures. The overall shape nevertheless agrees with the observed data. We also cannot exclude the influence of variations in the background.

The fitting process revealed a prominent ambiguity in the obtained best-fitting dust growth parameter sets. Comparing the sets, we found that they are based on the dominance of larger particles to create scattered light in the MIR. As a result, the images were not sensitive to the density threshold and the outer particles size, as long as the outer particles are smaller than $0.1 \mu \mathrm{m}$ to avoid substantial scattered light flux in the outer parts. This ambiguity is visible in the growth law defined in Eq. (1): changing the density threshold by a factor $f$ can be compensated for by adjusting the size of the outer grains by a factor $f^{\alpha}$ to get the same shape of the grain growth. In contrast, changing the spectral index had a strong effect on the images. Already for $\alpha=0.3$, the flux values were too low by a factor of 4 .

In general, the flux of the theoretical images is about a factor of 2 lower than the observed flux. We show the specific flux 


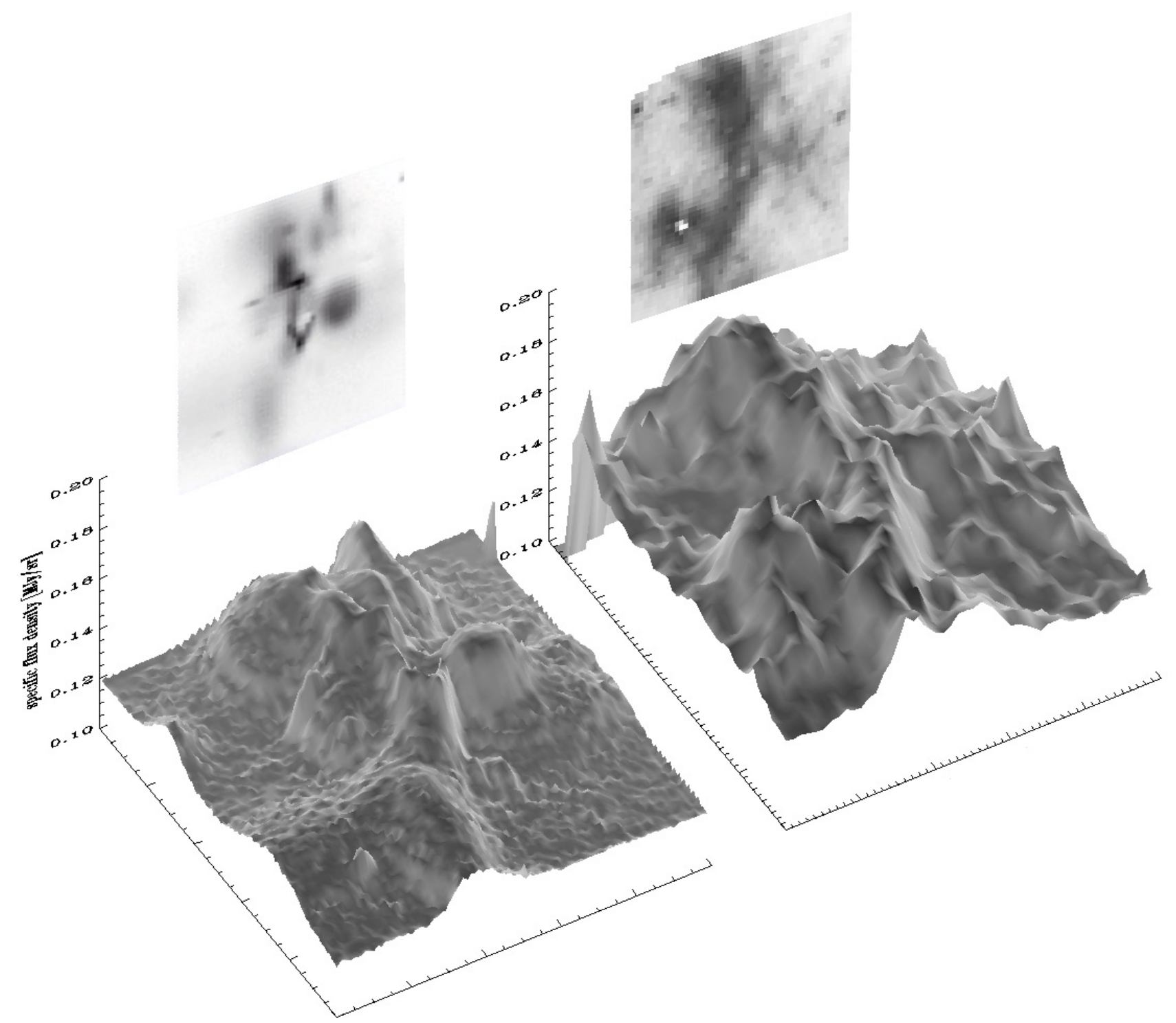

Fig. 9. Comparison of the specific flux density (z-axis) of the $3.6 \mu \mathrm{m}$ Spitzer image of the inner 66000 AU of L183 (right), processed through a star removal routine, with the scattered light model image (left).

density of model and observed image in Fig. 9 both as flat image and in surface representation to compare the actual values. As the stars confused the image substantially, we have processed the $3.6 \mu \mathrm{m}$ IRAC image through a routine that removes the stars. The routine considers the flux distribution of subimages and marks pixels with fluxes in the tail of the distribution, then interpolates them from the remaining pixels. The emission peak near the bright star in the lower left part of the image is an artefact from the spread-out PSF of this star.

While the flux pattern could be reproduced by the model, the higher flux could not be achieved by changing the grain properties or the spatial structure. Within the simple dust model, one might consider that higher flux value can be achieved by assuming a stronger growth of particles with the bigger grains producing more scattered light. The increased extinction, however, damps this radiation more efficiently so that the emission of these grains cannot be observed any more.

The flux will also change if we consider a more sophisticated dust model. As an example, if we consider elliptically shaped grains with perfect grain alignment to a large-scale magnetic field, the phase function, hence the scattered light flux, will increase by a factor of 2 by assuming a moderate grain main axes ratio of just 2 (see, e.g., Voshchinnikov \& Farafonov 1993).

It can be expected that an automated fitting and/or an improved modeling of the $A_{\mathrm{V}}$ map would remove most of the obvious peaked artefacts. Further improvement can be expected from improving the $A_{\mathrm{V}}$ map in the northern banana-shaped region because in the original study (Paper I), that part was not mapped with the MIR camera of the Infrared Space Observatory (ISOCAM). From mm measurements, the expected extinction reaches $A_{\mathrm{V}} \approx 60$ to 70 mag (being somewhat insecure for technical problems with the bolometer). This was beyond the sensitivity of the $H-K^{\prime}$ color-reddening method used to map this part to complement the ISOCAM map. Lower limits in the range $A_{\mathrm{V}}=25-40$ mag have been filled in instead, which did not induce dense clump fitting in the $3 \mathrm{D} A_{\mathrm{V}}$ model.

In a more realistic model, larger grains might be expected to grow as a second population from the original seed population. So there is the normal Rayleigh scattering by most particles and some more efficient scattering of bigger grains. In the simple 
model in the paper, all grains are moved away from the Rayleigh regime when growth occurs.

In test runs, we also estimated the limit for the largest grains we are able to detect with our method in L183 based on our dust model. For 3.6 and $4.5 \mu \mathrm{m}$, grains of about $1 \mu \mathrm{m}$ in size make the largest contribution to the scattered light flux. The flux from larger grains quickly drops because of the strong extinction within the dense regions.

An interesting point is the timescale of the growth process related to the evolution time scale of the core and cloud. If the clouds are long-lived, grains might grow enough to become a seed population for the subsequent planet formation process. The growth model that we adopted from Flower et al. (2005) calculated the growth status after one free-fall time. We will investigate this question further in a forth-coming paper dealing with the growth and maximal sizes expected for molecular cloud cores.

To sum up, we refer to the two goals formulated at the beginning of this section: (i) we have demonstrated that scattered light images based on a density structure that agrees with the current extinction data of L183 can reproduce the main features of the observed Spitzer images. Some deviations arise from numerical artefacts or incompleteness of the extinction data and are expected to be tackled with a better modeling; (ii) the presented images use a grain growth model, and indeed the images are very sensitive to the threshold density where growth starts, to the starting grain size, and to the growth efficiency. We find that models without dust growth do not produce any measurable scattered light flux, while a growth from grains with a size of about $0.05 \mu \mathrm{m}$ starting at an $\mathrm{H}_{2}$ number density of $3 \times 10^{10} \mathrm{~m}^{-3}$ can reproduce the emission pattern seen by Spitzer in the 3.6 and $4.5 \mu \mathrm{m}$ filter.

\section{Summary}

In this paper, we have presented Spitzer maps of the dark cloud complex L183 which reveal emission at 3.6 and $4.5 \mu \mathrm{m}$ and absorption at $8 \mu \mathrm{m}$ near the densest regions of the cloud $\left(A_{\mathrm{V}}>15\right)$. We argued that the emission cannot be attributed to thermal black body emission of larger dust grains, they are too cold in this environment. The emission of stochastically heated particles should originate in the layer where most of the interstellar radiation field is absorbed. The morphology of this layer is visible in the scattered light at optical wavelengths and traces the outer contours of the extinction map, not the inner banana-shaped region of L183. Moreover, existing PAH emission models show a strong increase in emission from 4.5 to $5.8 \mu \mathrm{m}$, which is not seen in the Spitzer images. Finally, a careful investigation of the density structure has confirmed that an excitation of stochastically heated particles (PAHs and small grains) through holes in the clumpy cloud is unlikely, because of the optical depth for the interstellar radiation at optical and UV wavelengths for almost all investigated spatial structure models for L183 (see Appendix A). We therefore suggested that this emission is scattered interstellar radiation.

To test this hypothesis, we performed 3D radiative transfer calculations producing scattered light images of the central dense region where emission is seen. The density structures were derived from an automated fit of the $A_{\mathrm{V}}$ map for this region (from Paper I) by a series of 3D Gaussian number density functions. The missing information along the line-of-sight was dealt with by creating a library of possible density structures in agreement with the $A_{\mathrm{V}}$ map.
When using simple silicate dust grains without growth, the produced scattered light flux was not sufficient to reproduce the observed image. This finding was independent of the chosen spatial structure and the dust grain size. The model included a realistic direction-dependent ISRF based on the DIRBE data, which did not introduce new unknowns that could change this view.

This led us to consider grain models that allow for grain growth in the denser parts of the cloud. Assuming a simple growth law along the directions of recent laboratory measurements, we investigated the changes in the optical depth and the scattering direction for photons propagating into regions with increasing density containing larger dust grains.

Within this model, we were able to reproduce the main features of the Spitzer observation in the 3.6, 4.5, and $8 \mu \mathrm{m}$ bands: (i) the emission arises mainly in the dense regions; (ii) the emission in the $4.5 \mu \mathrm{m}$ band is weaker than in the $3.6 \mu \mathrm{m}$ band and concentrated in the densest upper part of the banana-shaped central region; (iii) the central region appears in absorption at $8 \mu \mathrm{m}$. The flux is smaller by a factor of about 2 in the model images compared to the $3.6 \mu \mathrm{m}$ flux measured by Spitzer. However, various uncertainties enter the modeling, namely the uncertainties in the $A_{\mathrm{V}}$ map, the errors in the $A_{\mathrm{V}}$ map fitting, the uncertainties in the extinction properties of the dust, the uncertainty in the scattering phase function, the approximations in the dust model, and the uncertainty in the ISRF.

The existence of a new MIR window to study the growth of grains in the dense parts of cores is the result of an interplay of several effects: at MIR wavelengths, the moderate optical depth allows observing dense regions where growth is expected. The MIR scattering efficiency of smaller grains is too low to detect them, but larger grains scatter more efficiently and become observable again.

From our results we conclude that this is the reason why we see emission in the 3.6 and $4.5 \mu \mathrm{m}$ Spitzer images of L183 as interstellar radiation scattered by dust particles grown in the dense region with respect to common ISM particles. Therefore, the Spitzer observations of L183 are the first direct evidence of dust growth in molecular cloud cores. In turn, we evaluate the evidence from the extinction measurement at MIR and from FIR/mm observations to be indirect as there is an ambiguity in explaining the data by either assuming larger grains or by modifying the density and temperature structure used in the LoS integration.

In a future paper, we will present a $3 \mathrm{D}$ model of the dust based on a revised $A_{\mathrm{V}}$ map; and it will be strongly constrained by combining all available data from $\mathrm{mm}$ to NIR.

Acknowledgements. This research has made use of NASA's Astrophysics Data System Abstract Service. We are thankful to François Ménard, Nikolai Voshchinnikov, Thomas Henning, Cornelius Dullemond, and Roy van Boeckel for fruitful discussions and to Nicolas Flagey for his help with Spitzer data.

\section{Appendix A: Exploring the applicability of a PAH model: three-dimensional structure variations and $\tau$-iso-surfaces}

From the $A_{\mathrm{V}}$ map alone, it cannot be excluded that stellar emission is approaching the inner parts of L183 to excite PAHs at the locations where emission is seen in Spitzer images. Projection effects can hide holes which would allow such excitation, and would not to be unlikely given the clumpy structure of the core. Becausethe PAHs are excited by optical and UV photons, we 

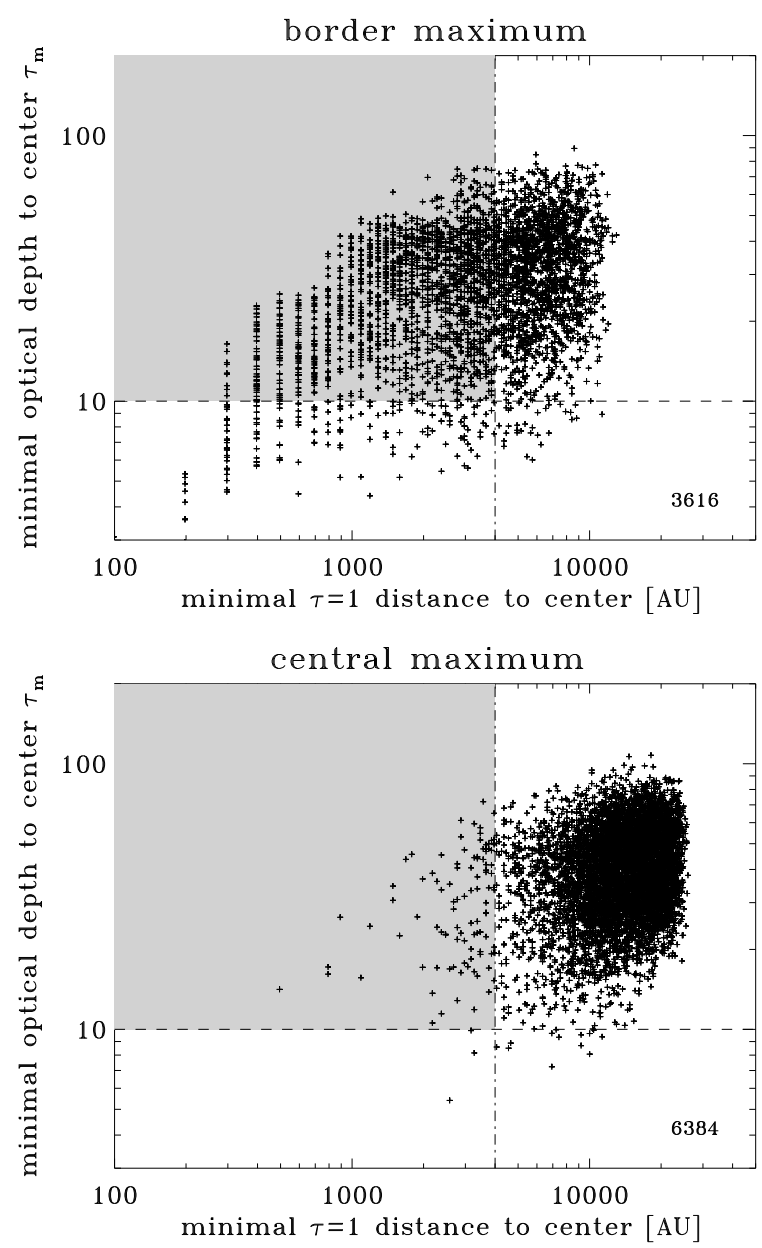

Fig. A.1. Minimal optical depth at $0.55 \mu \mathrm{m} \tau_{\mathrm{m}}$ to the central density maximum as a function of the 3D distance of the $\tau_{0.55 \mu m}=1$-surface to the central density maximum. The upper and lower plots show spatial structures for which the central density maximum is located within and outside the inner $30000 \mathrm{AU}$ of the spatial structure, respectively. The numbers in the lower right corner indicate the number of spatial structures in this category.

consider a wavelength of $0.55 \mu \mathrm{m}$ as a conservative limit if this radiation is not able to reach the PAHs then radiation at shorter wavelengths will not either. In this analysis, we define "reaching" as $\tau<1$. We performed spatial structure analysis calculations to explore the cases for which PAH excitation by the ISRF is possible. Varying the extent and position of the Gaussians along the line-of-sight randomly we obtained 10000 spatial structures, each of it in agreement with the L183 $A_{\mathrm{V}}$ map.

In the following, we would like to examine which of these spatial structures would allow for a PAH emission model. To characterize each configuration, we calculated the minimal $3 \mathrm{D}$ distance of the $\tau_{0.55 \mu \mathrm{m}}=1$-surface to the central density maximum $D_{\mathrm{m}}$ (as an indicator for density holes) and the minimal optical depth at $0.55 \mu \mathrm{m}$ toward the density maximum $\tau_{\mathrm{m}}$ (as indicator for the heating of the center by the ISRF). The results are plotted in Fig. A.1, which relates both quantities.

To distinguish the spatial structures with a central density maximum close to the front or back parts of the considered density cube (which is close to the low-density outer cloud part) and those that have the center in the middle part, we plotted those spatial structures in the upper (lower) panels for which the central density maximum is located within (outside) the inner $30000 \mathrm{AU}$ of the spatial structure, respectively. In the following, we refer to these two cases as central and border maximum spatial structures. Their numbers are given in the bottom right corner of each plot.

In the $3.6 \mu \mathrm{m}$ Spitzer image, emission is seen out to $D_{\mathrm{E}}=$ $4000 \mathrm{AU}$ measured in the plane of sky from the central density maximum. Assuming that the emission comes from the surface of a cylinder with this radius, the ISRF has to enter the cloud down to this distance to excite PAHs. The dash-dotted line indicates this distance $D_{\mathrm{E}}$ and the $\tau_{0.55 \mu \mathrm{m}}=1$-surface should be closer to the center or efficient PAH excitation will not be possible. As a conservative criterium, we assume that minimal distance of the $\tau_{0.55 \mu \mathrm{m}}=1$-surface to the center should be less than $D_{\mathrm{E}}$.

For most border maximum spatial structures and almost all central maximum spatial structures (right of the dash-dotted line) this is not the case. Exciting stellar optical light cannot reach the inner parts without being heavily extincted, so that $\mathrm{PAH}$ emission can be excluded as an explanation for the observed emission. A few border maximum spatial structures reach low $\tau_{\mathrm{m}}<10$ values. The resulting temperature of the central density peak would be higher than the 7-9 K found by Pagani et al. $(2003,2004)$. Thus, spatial structures below the dashed line at $\tau_{\mathrm{m}}<10$ are also not consistent with the observational data.

We are therefore left with spatial structures in the upper left hand corner of the two plots in Fig. A.1 to be candidates for possible PAH emission models. For the upper plot, the central density maximum can be located in the front part or in the back of the entire spatial structure. If it is located in the back, the excited PAH emission has to cross the entire spatial structure. Since $\tau_{3.6 \mu \mathrm{m}}$ is about an order of magnitude less than $\tau_{0.5 \mu \mathrm{m}}$, the expected optical depth would still be between a few to ten, making the PAH emission produced in the front part dominate the emission.

If it is located in the front, it will be illuminated well, hence expected to be seen in emission. Only if there is more flux created in the back by the other less dense clumps would it be seen in absorption. This region between the other clumps and the foreground clump are shielded well though, because to excite PAHs, radiation has to cross either the other clumps or the foreground clump.

Limb brightening in an optically thick clump would produce a ring of high surface brightness and a dip in the center. But such an external dense clump should be seen in the optical images of L183 as a small region with enhanced scattered light. But the CFHT I image only shows smooth scattered light on scales of the outer $\tau=1$ layer visible in the $A_{\mathrm{V}}$ map. Moreover, just a dip is seen but not a ring.

In view of these arguments, it appears unlikely that the central part of L183 has a density maximum located near the edge of the spatial structure. The remaining few central maximum spatial structures in the lower plot of Fig. A.1 left from the $D_{\mathrm{m}}$ dashdotted line and with $\tau_{\mathrm{m}}>10$ are the only spatial structures with holes arranged in a way that a PAH emission model would work.

With 10000 random spatial structures, a parameter space of 200 parameters is not well-sampled, but the main optical depth effects are dominated by a few ten Gaussians. From this analysis, we conclude that a PAH model for the $3.6 \mu \mathrm{m}$ emission cannot be excluded formally with the available data. From our sample of $3 \mathrm{D}$ spatial structures consistent with the $A_{\mathrm{V}}$ map, only a very small fraction would allow for PAH emission near the central absorption peak, which therefore seems unlikely. 


\section{Appendix B: A possible star within L183}

Beside the ISRF, another source of illumination could be the presence of a nearby star, positioned at $\sim 3^{\prime}$ away from the reference position. This star, visible in neither the blue POSS plates nor the red POSS I plate and hardly visible in the red POSS 2 plate, is bright in the infrared (Table B.1), implying that the star is attenuated by the cloud. We started by deriving a set of plausible reddening values (from the $H$ and $K^{\prime}$ difference, we found $A_{\mathrm{V}}$ in the range 10 to $23 \mathrm{mag}$ ). For this range, no normal star (main sequence, giant, supergiant) could fit the data.

We therefore applied a more detailed analysis, by applying an automated SED fitting procedure based on the simulated annealing code (Thamm et al. 1994) that calculates the spectrum using an extincted Planck spectrum varying the effective stellar surface temperature $T$, the distance, the stellar radius $R$, and the column density of extincting material for ISM Si and C grains. Figure B.1 shows the specific spectral energy density $\lambda F_{\lambda}$ as a function of $\lambda$. The data points are marked as thick crosses, no error bars have been considered as the given errors are small (see Table B.1). Comparing with an unextincted spectrum in the Rayleigh-Jeans limit (dotted line), no indication of IR excess is visible, which would arise from the thermal emission of warm dust in a nearby disk and/or shell.

We tried two ways to fit the spectrum with a stellar spectrum that undergoes no extinction. The dashed line gives a fit for a Planck spectrum that has the same maximum as the observed SED $\left(T_{*}=2400 \mathrm{~K}, R_{*}>3 \times 10^{6} \mathrm{~km}\right.$, same distance as L183). Cool object spectra fail to reproduce the sharp turnover around the maximum without extinction. The dash-dotted lines shows the Planck spectrum without extinction that follows the data where the deviation from the Rayleigh-Jeans spectrum occurs $\left(T_{*}=7000 \mathrm{~K}, R_{*}>2 \times 10^{6} \mathrm{~km}\right)$, but the flux decrease is too weak toward shorter wavelengths. Hotter objects cannot reproduce the sharp turnover either. We therefore concluded that extinction is needed to achieve better fits.

Putting a layer of extincting dust in front, we used the SED optimizer to investigate two cases: (i) silicate grains (solid thick line) and (ii) carbon grains (solid dash-three dotted line) (Draine $\&$ Lee 1984). The thick solid line gives the fitted spectrum for silicate grains, the dash-three dotted line for carbon grains. Both fits failed to reproduce the SED with good accuracy. In these models, the temperature was around $T_{*}=7000 \mathrm{~K}$, and the extinction was about $\tau=5$ to 9 depending on the dust model. It would need a more sophisticated dust and/or stellar atmosphere model to improve the fits. Given the projected position in the extinction map, the optical depth from the $A_{\mathrm{V}}$ map for the star when sitting behind the core would be 10 to 15 . This value is close to the extinction derived from the SED fitting. We conclude from these data that the star is not in front of the cloud.

We calculated the flux ratio of a star consistent with the SED fits at various distances from L183 and the ISRF at $3.6 \mu \mathrm{m}$ using DIRBE data. We find that the radiation field is dominated by the star up to about a distance of $2000 \mathrm{AU}$ and then the stellar influence drops quickly due to the quadratical decrease with distance. If the star were placed near L183 or even exactly at the same distance to Earth as the density maximum, it still would be located in the outer parts of L183 as is evident in Fig. 1, and we would only expect a very local (a few thousand AU) influence on the scattered light pattern, while the ISRF dominates for the entire banana-shaped region. The potential influence of the star being placed near L183 is weakened further by its having to be near a dense region with grown particles to produce scattered light at 3.6 micron.
Table B.1. Star position and magnitudes.

\begin{tabular}{cccc}
\hline \hline $\begin{array}{c}\text { Position } \\
(\mathrm{J} 2000)\end{array}$ & $\begin{array}{c}\text { RA } \\
15^{\mathrm{h}} 54^{\mathrm{m}} 20.45^{\mathrm{s}}\end{array}$ & $\begin{array}{c}\text { Dec } \\
-02^{\circ} 54^{\prime} 07.30^{\prime \prime}\end{array}$ \\
\hline $\begin{array}{c}\text { Band } \\
(\mu \mathrm{m})\end{array}$ & $\begin{array}{c}\text { Brightness } \\
\text { mag }\end{array}$ & $\begin{array}{c}\text { Error } \\
\mathrm{mag}\end{array}$ & Ref. \\
\hline$R$ & $(19.7)^{a}$ & & 1 \\
$I$ & 16.3 & 0.1 & 2 \\
$J$ & 10.986 & 0.021 & 3 \\
$H$ & 8.915 & 0.044 & 3 \\
$K$ & 7.910 & 0.016 & 3 \\
3.6 & 7.361 & 0.002 & 4 \\
4.5 & 7.340 & 0.002 & 4 \\
5.8 & 7.222 & 0.003 & 4 \\
8 & 7.177 & 0.003 & 4 \\
24 & 7.4 & 0.1 & 4 \\
\hline
\end{tabular}

Notes. ${ }^{(a)}$ USNO-B calibrations are done in the original $E$ band plate, which differs somewhat from the standard $R$ band (Monet et al. 2003).

References. (1) USNO-B (Monet et al. 2003); (2) unpublished data from Paper I; (3) 2MASS (Cutri et al. 2000); (4) this work.

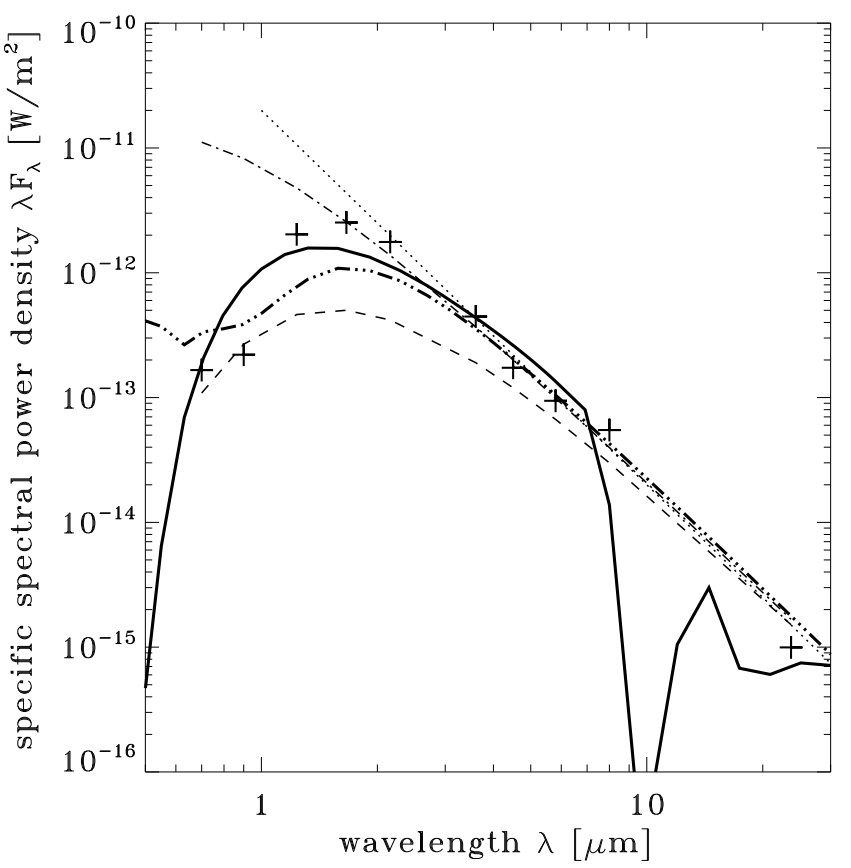

Fig. B.1. Specific spectral energy density as a function of the wavelength. The observed data points are shown as thick crosses. Three fits are displayed without extinction are displayed: a spectrum in the Rayleigh-Jeans limit (dotted), a Planck spectrum having the same maximum as the observed SED (dashed), and a Planck spectrum that follows the data where the deviation from the Rayleigh-Jeans spectrum occurs (dashed-dotted). For the case of extincted stellar emission, two spectra are shown: a Planck spectrum of a star being extincted by silicate grains (thick solid), and by carbon grains (dash-three dotted), respectively.

\section{References}

Beuther, H., Hunter, T. R., Zhang, Q., et al. 2004, ApJ, 616, L23 Chapman, N. L., \& Mundy, L. G. 2009, ApJ, 699, 1866

Chapman, N. L., Mundy, L. G., Lai, S.-P., \& Evans, N. J. 2009, ApJ, 690, 496 
Cutri, R. M., Skrutskie, M. F., Van Dyk, S., et al. 2000, Explanatory Supplement to the 2MASS Second Incremental Data Release, Tech. rep., IPAC/Caltech

Draine, B. T. 2003a, ARA\&A, 41, 241

Draine, B. T. 2003b, ApJ, 598, 1017

Draine, B. T. 2003c, ApJ, 598, 1026

Draine, B. T., \& Lee, H. M. 1984, ApJ, 285, 89

Draine, B. T., \& Li, A. 2007, ApJ, 657, 810

Fazio, G. G., Hora, J. L., Allen, L. E., et al. 2004, ApJS, 154, 10

Flagey, N., Boulanger, F., Verstraete, L., et al. 2006, A\&A, 453, 969

Flower, D. R., Pineau Des Forêts, G., \& Walmsley, C. M. 2005, A\&A, 436, 933

Foster, J. B., \& Goodman, A. A. 2006, ApJ, 636, L105

Franco, G. A. P. 1989, A\&A, 223, 313

Juvela, M., Mattila, K., Lehtinen, K., et al. 2002, A\&A, 382, 583

Juvela, M., Pelkonen, V.-M., Padoan, P., \& Mattila, K. 2008, A\&A, 480, 445

Kiss, C., Ábrahám, P., Laureijs, R. J., Moór, A., \& Birkmann, S. M. 2006, MNRAS, 373, 1213

Makovoz, D., \& Marleau, F. R. 2005, PASP, 117, 1113

McClure, M. 2009, ApJ, 693, L81

Monet, D. G., Levine, S. E., Canzian, B., et al. 2003, AJ, 125, 984

Nakajima, Y., Nagata, T., Sato, S., et al. 2003, AJ, 125, 1407
Nutter, D., Kirk, J. M., Stamatellos, D., \& Ward-Thompson, D. 2008, MNRAS, 384,755

Ossenkopf, V. 1993, A\&A, 280, 617

Ossenkopf, V., \& Henning, T. 1994, A\&A, 291, 943

Padoan, P., Juvela, M., \& Pelkonen, V.-M. 2006, ApJ, 636, L101

Pagani, L., Lagache, G., Bacmann, A., et al. 2003, A\&A, 406, L59

Pagani, L., Bacmann, A., Motte, F., et al. 2004, A\&A, 417, 605

Pagani, L., Bacmann, A., Cabrit, S., \& Vastel, C. 2007, A\&A, 467, 179

Poppe, T., \& Blum, J. 1997, Adv. Space Res., 20, 1595

Ridderstad, M., Juvela, M., Lehtinen, K., Lemke, D., \& Liljeström, T. 2006, A\&A, 451, 961

Sandell, G., \& Mattila, K. 1975, A\&A, 42, 357

Schnee, S., Li, J., Goodman, A. A., \& Sargent, A. I. 2008, ApJ, 684, 1228

Steinacker, J., Henning, T., Bacmann, A., \& Semenov, D. 2003, A\&A, 401, 405

Steinacker, J., Bacmann, A., Henning, T., Klessen, R., \& Stickel, M. 2005, A\&A, 434, 167

Steinacker, J., Chini, R., Nielbock, M., et al. 2006, A\&A, 456, 1013

Stepnik, B., Abergel, A., Bernard, J.-P., et al. 2003, A\&A, 398, 551

Thamm, E., Steinacker, J., \& Henning, T. 1994, A\&A, 287, 493

Voshchinnikov, N. V., \& Farafonov, V. G. 1993, Ap\&SS, 204, 19 